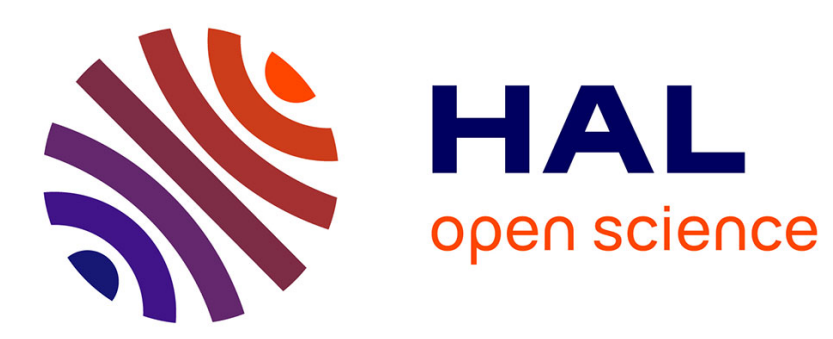

\title{
Sending more with less: crowdsourcing integrated transportation as a new form of citywide passenger-package delivery system
}

Chao Chen, Daqing Zhang, Zhu Wang

\section{- To cite this version:}

Chao Chen, Daqing Zhang, Zhu Wang. Sending more with less: crowdsourcing integrated transportation as a new form of citywide passenger-package delivery system. IT Professional, 2020, 22 (1), pp.56-62. 10.1109/MITP.2018.2887389 . hal-03363366

\section{HAL Id: hal-03363366 https://hal.science/hal-03363366}

Submitted on 30 Jan 2022

HAL is a multi-disciplinary open access archive for the deposit and dissemination of scientific research documents, whether they are published or not. The documents may come from teaching and research institutions in France or abroad, or from public or private research centers.
L'archive ouverte pluridisciplinaire HAL, est destinée au dépôt et à la diffusion de documents scientifiques de niveau recherche, publiés ou non, émanant des établissements d'enseignement et de recherche français ou étrangers, des laboratoires publics ou privés. 


\section{Feature Article}
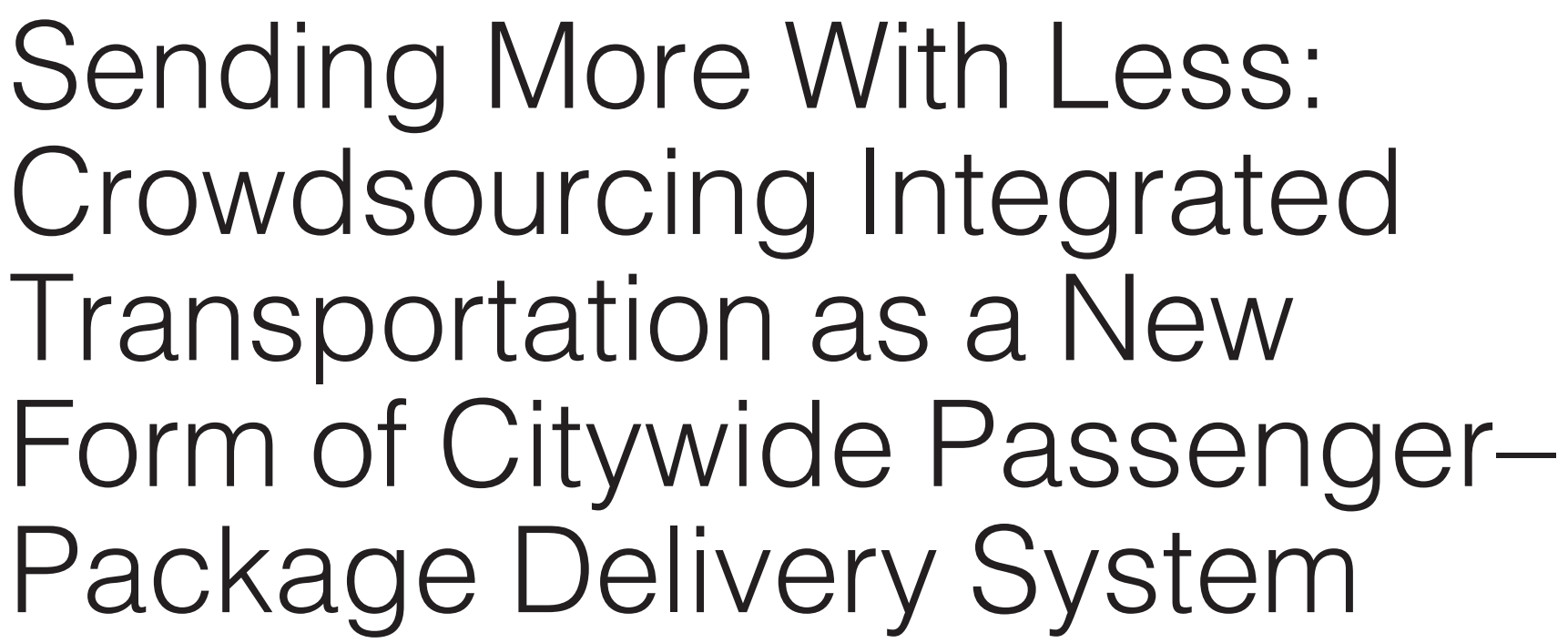

Chao Chen

Chongqing University

Zhu Wang

Northwestern Polytechnical University
Daqing Zhang

Telecom SudParis

Abstract-Although much effort has been devoted by both academic and industrial communities to improve the efficiency of urban passenger and package flows, current urban transport systems still fail to balance the speed and cost. To fill the gap, in this paper, we propose a novel form of transport system called crowdsourcing integrated transportation (CIT). It leverages the underused transport capacity which is generated while delivering passengers to hitchhike packages so that more transportation needs can be met with fewer vehicles and drivers (i.e., sending more with less). We identify the unique features of the new delivery system when comparing to the traditional transport systems and discuss the key research challenges and potential solutions. We further implement the passenger-occupied taxis as the package carriers and evaluate the effectiveness. With several future research directions discussed in $\mathrm{CIT}$, we expect that more research interests will be stimulated in this novel transport paradigm.

People and goods are the two most important elements of city components. ${ }^{1,2}$ How to

Digital Object Identifier 10.1109/MITP.2018.2887389

Date of current version 12 February 2020. distribute them in a cost-effective way around the city has become a long-standing research issue from both academic and industrial communities. ${ }^{3}$ The issue becomes even more urgent yet vital in the context of the increasing urbanization when increasingly more people are gathering in 
the city, and the exponential growth of package delivery demands result from the sustainable development of E-commerce. ${ }^{4}$

Speed and cost are two major concerns when transporting people and packages; however, they generally conflict each other. ${ }^{4,5}$ More precisely, the improvement on the speed usually indicates the increase of vehicle number and the delivery frequency, as a result, the increase of the transport cost. To make matter worse, more serious environmental and social problems can also be generated, since the transportation produces significant negative externalities, such as carbon footprint, traffic accidents, and congestions. Therefore, how to design an innovation city-wide transport system that can better balance the two conflicting objectives has been intensifying in many cities and countries. ${ }^{6}$

Thanks to the advancement in information and communication technologies, especially the wide prevalence of the global position system (GPS) and mobile Internet in recent years, a large number of vehicles, such as taxis, public buses, logistics vans, and trucks, have been equipped with GPS devices and continuously send their real-time positions and travel status (e.g., origins, intended destinations, available capacity) to the server. ${ }^{6,7}$ Such developments open up new opportunities to cope with the increasingly growing transport demands in the inexpensive fashion. Quite a few of technological solutions are provided, including the following.

- To passengers, ride-hailing platforms are gradually becoming mature that enable the timely and efficient supply-and-demand matching than ever before. ${ }^{8,9}$

- The idea of ride-sharing (e.g., carpooling and carsharing) has been widely applied in people's daily life to increase the passengers' mobility but with smaller fleet of vehicles when transporting passengers, making a considerable contribution to the sharing economy. ${ }^{10}$

- Crowdsourcing logistics (also known as crowdsourced logistics or crowdshipping), which can be simply viewed as "applying the concept of crowdsourcing dedicatedly to the field of urban logistics," has been recognized as a powerful supplement to current urban goods delivery systems. ${ }^{11,12}$ A number of commercial and Internet giants, including DHL,
Walmart, Amazon, and several startups in industry, have launched the new services, but many important technical details (e.g., how to dispatch packages, how to fulfill the route planning tasks) are missing. Refer to the work presented by McKinnon et al. ${ }^{11,13}$ for a completed list of companies, apps, services, and other related information.

Currently, sending passengers and packages are often handled (or optimized) separately, although passenger flow and package flow actually have the sufficient overlap, e.g., share a number of city infrastructures, the basic principles of optimization. In this paper, to fill the gap, we thus propose the idea of sending more with less by hitchhiking packages with travelling passengers and fully utilizing the extra loading capacity. More specifically, we propose the system of crowdsourcing integrated transportation (CIT) - a new form of city-wide delivery system, which integrates the passenger and package flows based on the existing mobility pattern and infrastructure while reducing traffic congestion, pollution, and costs.

In this paper, to the best of our knowledge, we are among the pioneers to introduce the notion of CIT systematically, mainly from a perspective of the data scientist. First, we briefly overview a generic framework of CIT, followed by the unique characteristics. Second, we make a brief summary of our practice on CIT by highlighting the working procedure and some experimental results. Third, we identify the key research challenges and discuss some potential solutions. Finally, we discuss the practical issues and major obstacles from the various involved stakeholders in CIT before truly realizing the system, and conclude the work with some future research directions.

\section{GENERIC FRAMEWORK AND UNIQUE CHARACTERISTICS OF CIT}

\section{Generic Framework}

CIT mainly consists of three components: crowdsourced resources, package delivery tasks, and online platform, as shown in Figure 1. Crowdsourced resources are dedicatedly called to send passengers from their origins to the destinations simultaneously; they are also available for providing hitchhiking rides using the 


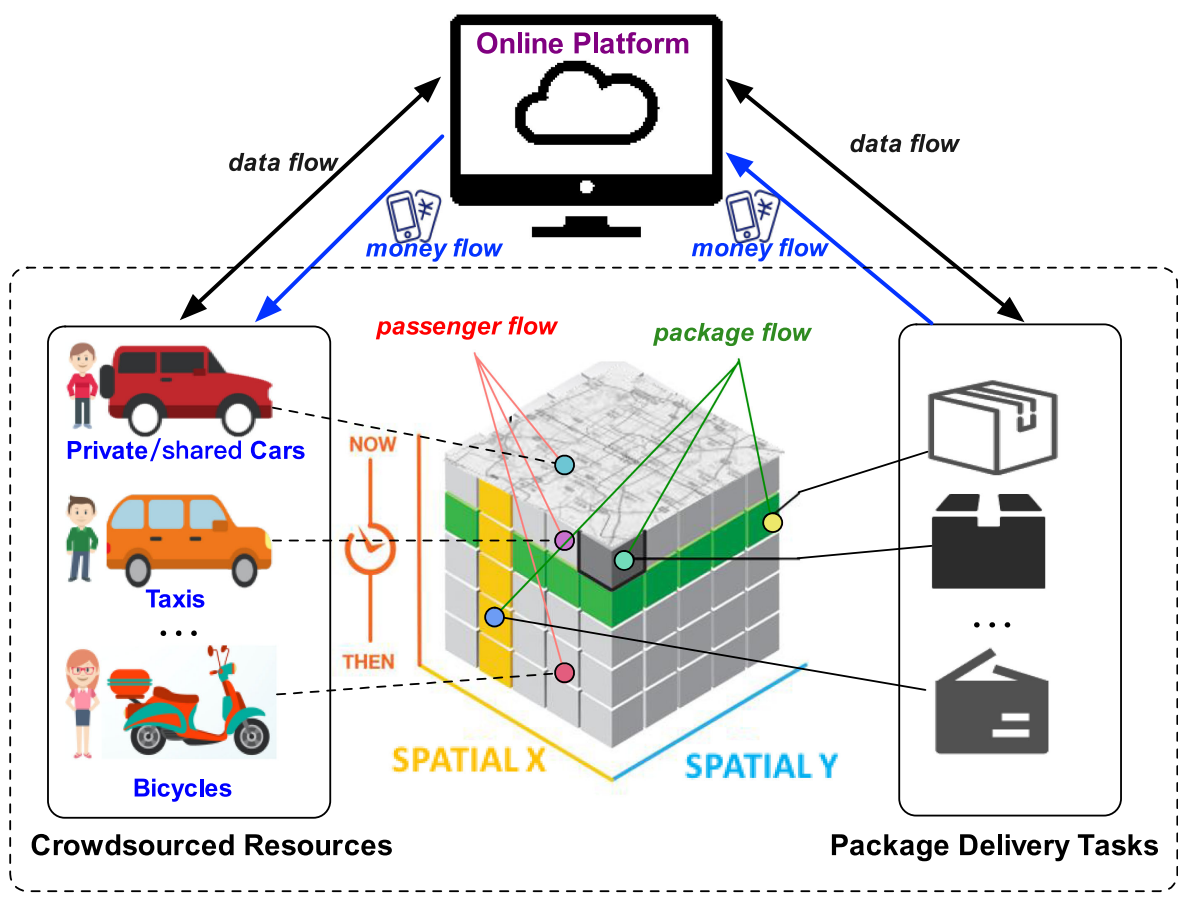

Figure 1. Overview of the generic framework of CIT. There are four kinds of flows, including data flow, passenger flow, package flow, and money flow, which are communicated within CIT.

spare capacity to complete the package delivery tasks. More precisely, from the origin to the destination, people may drive cars (shared or owned privately), take taxis, ride motor-cycles, and so on. At the same time, they are creating spare capacity for hitchhiking packages. However, the optimization of passenger flow and parcel flow integration (i.e., the dispatch and scheduling of crowdsourced resources for package deliveries) is challenging. Thanks to the development of the mobile Internet and positioning technology, the real-time location and time information in a fine resolution about passenger flows and package flows can be easily recorded and communicated (the red and green solid lines in Figure 1), providing rich data sources to solve the issue. With the received data flows and the embedded intelligent algorith$\mathrm{ms}$, the online platform component bridges the crowdsourced resources and package delivery task components and further transforms the data into decisions. The money flows from package senders to the crowdsourced drivers after accomplishing tasks as incentives, again via the online platform (the blue solid line in Figure 1). In summary, the system of CIT is an integrator of four kinds of flows, i.e., passenger, package, data, and money flows, among which the data flow is the key enabler.

\section{Unique Characteristics}

We highlight the unique characteristics of CIT by comparing it to the traditional urban package delivery systems.

- Flexibility: Compared to the current solutions to the city logistics that collect and distribute packages at most twice a day (i.e., customers can only send/receive packages at the appointed time windows specified by logistics companies), for consolidation purposes, CIT offers a flexible time schedule to customers since the package delivery is processed, managed, and distributed individually, according to the customer's request.

- Fluidity: The package delivery time is the sum of the waiting time for the postman to collect and the travel time on the roads from its origin to the destination. With CIT, on one hand, relatively less waiting time is necessary since the recruitment of vehicles is invoked immediately after the package delivery request is generated. On the other hand, less travel time is spent on the roads since the recruited 
vehicles usually deliver passengers fast by choosing light-traffic routes from an origin to a destination. However, for traditional urban delivery systems, in addition to the time consolidation, packages can only be sent from a few of satellite facilities or distribution centers that are far away from city centers before sending to the final customers, incurring unnecessary travel distance and time.

- Friendliness: By involving regular road users in the pickup and delivery process, CIT not just significantly reduces the transportation costs but also makes a significant contribution to the alleviation of environmental problems in terms of air quality (especially NOx and PM10), noises, and congestions. Meanwhile, social problems in terms of time-intensive laborious and heavy workloads by the consolidation of transport volumes per trip can also be alleviated.

- Fairness: The shipping price is a frequent obstacle not only to online shoppers but to small brick-and-mortar retails as well. Moreover, due to high shipping costs, providing home-delivery service is becoming a privilege of giant E-commerce companies in the current supply-chain system. Consequently, an increasing number of customers are attracted to buy things online, which threatens the growing of small brick-and-mortar retails. Fortunately, CIT can offer a fair price and access to any users, independent of the company size and location, to make them more competitive.

\section{OUR PRACTICE: USING}

PASSENGER-OCCUPIED TAXIS AS PACKAGE HITCHHIKERS

Taxis have become one of the main transportation means for commuting and travel in modern cities, generating abundant hitchhiking rides for package deliveries in the city. Thus, in our practice, we use passenger-occupied taxis as package hitchhikers. ${ }^{4}$ However, it should be noted that exploiting taxis with passengers to ship packages is often more complicated than $a$ simple query matching. ${ }^{5}$ For instance, due to financial considerations, few passengers will choose to take taxis if the destination is too far. In this case, a single hitchhiking ride may not be able to deliver a distant package to its destination; instead, a relay of taxis is needed.

Hence, we formulate the taxi-based package delivery as a route planning problem (i.e., finding the station combinations and scheduling taxi) with the objective of minimizing the package delivery time and have proposed a two-phase framework called CrowdDeliver to find the near-optimal delivering route for each package delivery task. CrowdDeliver consists of three major components, i.e., data preprocessing, offline trajectory data mining, and online package routing. The data preprocessing component is responsible for identifying citywide package interchange stations. They serve the functions of collecting, storing, and interchanging packages. The offline trajectory data mining component is mainly responsible for estimating the time-dependent package travel time between any two package stations by mining the historical taxi trajectory data. On top of such estimated time, for a package delivery task, we are able to discover an optimal route with the estimated delivery time in the statistical sense. The component of online package routing is designed to overcome the stochastic and uncertainty features of both passenger rides and package delivery tasks, by adaptively package rerouting and adaptive taxi scheduling according to the real-time information about taxi trajectory. See the work presented by Chen et al. ${ }^{4}$ for more technical details.

We have evaluated the proposed framework using the real-world datasets, i.e., the POI data, the road network data, and one month of taxi trajectory data generated by 7614 taxis in the city of Hangzhou, China. We have selected 852 package stations in total near the road sides around the whole city. For the package delivery tasks, we randomly generate its birth time, origin, and destination. We also report the success rate and delivery time (mean and standard deviation) with respect to the time of the day (i.e., day time and night time) and day of week (i.e., work day and rest day) in Figure 2. During the day, the packages are easier to deliver on work days (with a higher success rate and smaller package delivery time) than on other days. The result is the opposite at night. One possible reason is that most people tend to stay at home after work for a good rest. In contrast, they are more likely to go out for fun in the evening during rest days. 


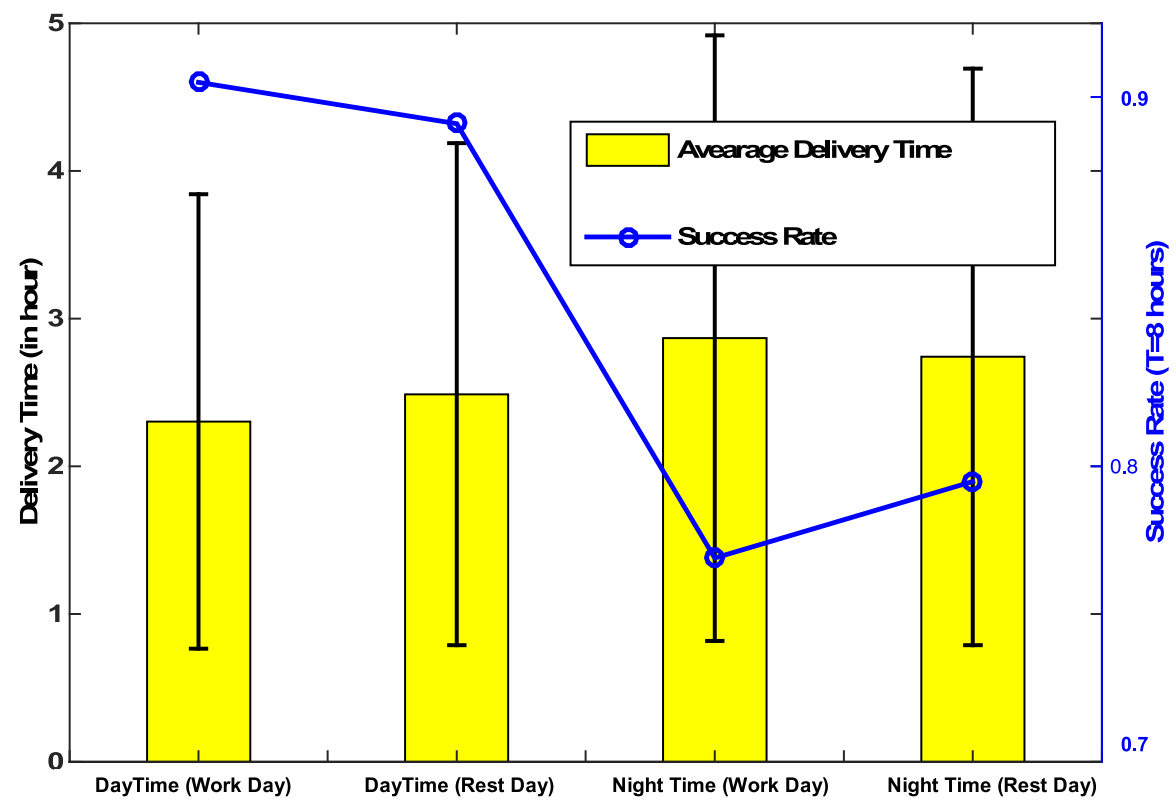

Figure 2. Results of success rate and package delivery time under different time slots.

\section{KEY RESEARCH CHALLENGES AND POTENTIAL SOLUTIONS}

The new system of integrated passengerpackage delivery also raises new research challenges and issues, some of which are discussed as follows.

- Passenger-package ridesharing is challenging: Although few techniques have been proposed to organize and manage passengers with multiple origins and destinations in a shared space, they cannot be applied to the passenger-package sharing directly for the following reasons.

1) The waiting time of the passenger-package sharing is much more flexible than passenger-passenger sharing. Nobody would accept the ridesharing service if they have to wait too long.

2) Unlike passengers who can proactively take part in ridesharing (e.g., walking to the appointed places and getting on cars by themselves), packages can only be uploaded/offloaded manually and passively.

3) In addition, passenger flow and package flow mismatch in both time and space, which further complicates the ridesharing between passengers and packages.
Compared to the passenger flow, little research attention has been taken to the spatio-temporal patterns of the package flow, probably due to the limited availability of the related data. ${ }^{14}$ To migrate the mismatch issue, one potential solution is the introduction of interchange stations and an increase in the degree of spatial and temporal matching between passenger and package flows (i.e., the indirect matching). Moreover, interchange stations also facilitate the temporary package storing, which may potentially increase the usability of crowdsourced resources. ${ }^{4}$

- Fine-grained citywide passenger flow forecasting is nontrivial: Citywide passenger flow prediction in a fine spatial and temporal resolution is one of the useful tools to know when and where the passenger will be originated and ended in advance, which is vital for the scheduling of package-hitchhiking rides. However, such a forecasting task is difficult and remains unsolved due to the uncertain and stochastic characteristics of people movement. A number of time-series predictors have been successfully used, including predictors based on deep models outperform in most cases. In more detail, the recurrent neural network and its variant long-short term memory are widely 
adopted since they are capable of capturing regular temporal patterns, as well as dynamic temporal patterns. ${ }^{15}$ Besides, passenger flow between two areas is highly affected by the nearby spatial context; the convolution neural network is thus integrated to achieve high accuracy. ${ }^{16}$ However, the design of a deep model is usually labor intensive, and model redesign is required when applying scenarios are changed. To make matters worse, deep models lack the interpretability and are hard to understand. In contrast, designing interpretably linear models by feeding high-level features is promising and worth being explored further. ${ }^{17}$ One potential way to derive high-level features is the combination of basic features extracted from multisource urban datasets. This feature can quantify the correlations among different basic features, and the descriptive capacity of the linear models can be enhanced.

- Package routing optimization in the real-time setting is difficult: For a package delivery task, if the information about the passenger flows after the time of the package delivery request are all known in advance, it is trivial to discover its "optimal" route (e.g., with the minimal time cost) by computing and comparing the total package delivery time of each possible delivery route. Such a problem belongs to the family of the deterministic optimization. However, in real-life applications, the stochastic, dynamics, and uncertainty of passenger and package flows increase the complexity of the package routing problem. Under such circumstances, traditional deterministic optimization algorithms may not be suitable to figure out the optimal delivery route. ${ }^{18}$ Therefore, novel and intelligent optimization algorithms that can adaptively schedule the crowdsourced resources according to the information about the real-time passenger and package flows are a necessity.

\section{DISCUSSION AND CONCLUSION}

Although integrating passenger and package flows demonstrates the potential to lead to more efficient and sustainable city operations since the same transportation needs can be met using fewer vehicles and drivers, there are still many issues to be further addressed before realizing the concept of CIT. To name a few, the human mobility is a key issue since the CIT intends to send packages and passengers in a shared space, making the transport synergy between them important. On one hand, human mobility becomes more complicated since new options (e.g., self-driving) for people travelling are emerging. On the other hand, more package delivery choices are provided in order to cope with the increasing demands for the speedy and timely delivery, making the integration of the two kinds of flows even more challenging. Last but not the least, the feasibility is essential to make CIT become a reality. Since CIT makes the use of passenger-delivery rides to hitchhike packages, a key consideration should be that the negative effect on the service for passengers is insignificant. Moreover, many other factors, including regulations from local government, public acceptance, rewarding mechanisms (pricing and incentive), the bidirectional trust and responsibility, and the business model, may hinder real-life implementations.

In this paper, a novel urban transportation system named CIT is proposed to significantly reduce transportation resources while still meeting travel demands of both passengers and packages (i.e., sending more with less). The rationale behind CIT is the fact that many transport capacities are underused while sending passengers and can be a rich resource for package deliveries. Our practice of CIT is also discussed to demonstrate the effectiveness of the proposed ideas and techniques. In the future, practical issues in CIT, such as user mobility, scalability, and feasibility, need to be further investigated.

\section{ACKNOWLEDGMENTS}

This work was supported in part by the National Key Research and Development Project of China (2017YFB1002000), in part by the National Natural Science Foundation of China (61602067 and 61872050), in part by the Fundamental Research Funds for the Central Universities (2018cdqyjsj0024), and in part by the Chongqing Basic and Frontier Research Program (cstc2018jcyjAX0551). C. Chen is the corresponding author for this paper. 


\section{REFERENCE}

1. C. Chen, Z. Wang, and B. Guo, "The road to the Chinese smart city: Progress, challenges, and future directions," ITProfessional, vol. 18, no. 1, pp. 14-17, 2016.

2. L. Wang, B. Guo, and Q. Yang, "Smart city development with urban transfer learning," IEEE Comput., 2018, to be published, doi: 10.1109/MC.2018.2880015.

3. M. Savelsbergh and T. Van Woensel, "City logistics: Challenges and opportunities," Transp. Sci., vol. 50, no. 2, pp. 579-590, 2016.

4. C. Chen et al., "Crowddeliver: Planning city-wide package delivery paths leveraging the crowd of taxis," IEEE Trans. Intell. Transp. Syst., vol. 18, no. 6, pp. 1478-1496, Jun. 2017.

5. C. Macharis and B. Kin, "The 4 A's of sustainable city distribution: Innovative solutions and challenges ahead," Int. J. Sustain. Transp., vol. 11, no. 2, pp. 59-71, 2017.

6. W. Lerner and F.-J. Van Audenhove, "The future of urban mobility: Towards networked, multimodal cities in 2050," Public Transport Int. English Ed., vol. 61, no. 2, pp. 14-18, 2012.

7. P. S. Castro, D. Zhang, C. Chen, S. Li, and G. Pan, "From taxi GPS traces to social and community dynamics: A survey," ACM Comput. Surveys, vol. 17, pp. 17:1-17:34, 2013.

8. L. Rayle, D. Dai, N. Chan, R. Cervero, and S. Shaheen, "Just a better taxi? A survey-based comparison of taxis, transit, and ridesourcing services in San Francisco," Transport Policy, vol. 45, pp. 68-178, 2016.

9. C. Chen, S. Jiao, S. Zhang, W. Liu, L. Feng, and Y. Wang, "Tripimputor: Real-time imputing taxi trip purpose leveraging multi-sourced urban data," IEEE Trans. Intell. Transp. Syst., vol. 19, no. 10, pp. 3292-3304, Oct. 2018.

10. L. Einav, C. Farronato, and J. D. Levin, "Peer-to-peer markets," Annu. Rev. Econ., vol. 8, no. 1, pp. 615-635, 2016.

11. A. C. McKinnon, "Crowdshipping: A communal approach to reducing urban traffic levels?" Kuehne Logistics Univ., Hamburg, Germany, Tech. Rep., 2016, doi: 10.13140/RG.2.2.20271.53925

12. H. Paloheimo, M. Lettenmeier, and H. Waris, "Transport reduction by crowdsourced deliveries-A library case in Finland," J. Cleaner Prod., vol. 132, pp. 240-251, 2016.
13. J.-F. Rougèsa and B. Montreuil, "Crowdsourcing delivery: New interconnected business models to reinvent delivery," in Proc. Int. Physical Internet Conf., 2014, pp. 28-30.

14. X. Tan, Y. Shu, X. Lu, P. Cheng, and J. Chen, "Characterizing and modeling package dynamics in express shipping service network," in Proc. IEEE Congr. Big Data, 2014, pp. 144-151.

15. J. Zhang, Y. Zheng, D. Qi, R. Li, X. Yi, and T. Li, "Predicting citywide crowd flows using deep spatiotemporal residual networks," Artif. Intell., vol. 259, pp. 147-166, 2018.

16. H. Yao et al., "Deep multi-view spatial-temporal network for taxi demand prediction," in Proc. AAAl Conf. Artif. Intell., 2018, pp. 2588-2595

17. Y. Tong et al., "The simpler the better: A unified approach to predicting original taxi demands on largescale online platforms," in Proc. ACM SIGKDD Conf. Knowl. Discovery Data Mining, 2017, pp. 1653-1662.

18. A. M. Arslan, N. Agatz, L. Kroon, and R. Zuidwijk, "Crowdsourced delivery—A dynamic pickup and delivery problem with ad hoc drivers," Transp. Sci., 2018, to be published, doi: 10.1287/trsc.2017.0803.

Chao Chen is a full professor of computer science at Chongqing University, China. His research interests include pervasive computing, social network analysis, mobile crowdsensing, green logistics, and big data analytics for smart city applications. Contact him at cschaochen@cqu.edu.cn.

Zhu Wang is an associate professor of computer science at Northwestern Polytechnical University, China. His research interests include pervasive computing, social network analysis, community detection, and mobile healthcare. Contact him at wangzhu@nwpu.edu.cn.

Daqing Zhang is a professor of computer science at SAMOVAR and Telecom SudPairs, France. His research interests include large-scale data mining, urban computing, context-aware computing, and ambient assistive living. Contact him at daqing. zhang@telecom-sudparis.eu. 